\title{
Model and Validation of a Highly Extensible and Tough Actuator based on a Ballooning Membrane
}

\author{
Nicolas Herzig ${ }^{1,2}$, Joanna Jones ${ }^{1}$, Eduardo Perez-Guagnelli ${ }^{1}$, and Dana D. Damian ${ }^{1}$
}

\begin{abstract}
Soft robots are known for their ability to comply and having superior extensibility. However, one of the limitations of most of these robots is that they can stand only a limited amount of load before buckling, and they feature a non-negligible initial height. Hybrid soft-rigid actuators seem to offer a trade-off between compliance and the amount of load they can withstand, but only a few simple models have been proposed to describe the behavior of these actuators. In this paper, we propose a design, model and experimental validation of a Hyperelastic Ballooning Membrane actuator (HBMA) which shows an extensibility higher than $179 \%$, as well as an ability to stand more than 20 times its own weight. Two models, giving the dynamic behavior of the HBMA in terms of displacement and pressure, have been derived from different hyperelastic models (Neo-Hookean and Mooney-Rivlin) and compared in terms of accuracy and robustness. Finally, an example of a hybrid soft-rigid continuum ballooning robot built with HBMAs is presented and characterized experimentally.
\end{abstract}

\section{INTRODUCTION}

A soft robotics approach has been widely applied to the medical field with applications varying from haptics and wearables [1], to minimally invasive surgery and implants [2], [3], as well as assistive technology [4]. By being highly compliant, soft robots have proved themselves suitable for applications inside and outside the body, by allowing safe interactions and being conformable. In particular, the development of robotic implants that exploit soft materials seems promising to reduce foreign body response and inflammation [5]. Robotic implants require to be extensible while they apply a force to induce the growth of the tissue they are attached to [2], [6], [7]. In cases where large portions of the tissue are missing, such as short bowel syndrome and long gap oesophageal atresia, the need for soft actuators capable of large extensions with low initial volume is crucial [8], [9].

Soft actuators extending around $100 \%$ have already been demonstrated [10] [11], but require a considerable initial length of actuator to be extended. Moreover, these typical soft actuators are susceptible to buckling with larger deformations. For in vivo operations, buckling of the extensible actuator leads to the risk of obstructing the food flow of tubular tissues that the actuator is lengthening.

In this work, we propose extensible robots based on membrane actuators. These have the advantage of having large growth through ballooning when stacked, as well as being

This work was funded by The United Kingdom Engineering and Physical Sciences Research Council (EPSRC) Tissue-RIMOTE grant [EP/S021035/1]

1 Department of Automatic Control and Systems Engineering, the University of Sheffield, S1 3JD Sheffield, United Kingdom. d.damianesheffield.ac.uk

2 Department of Engineering and Design, University of Sussex, BN1 9RH, Brighton, United Kingdom. n.f.herzig@sussex.ac.uk

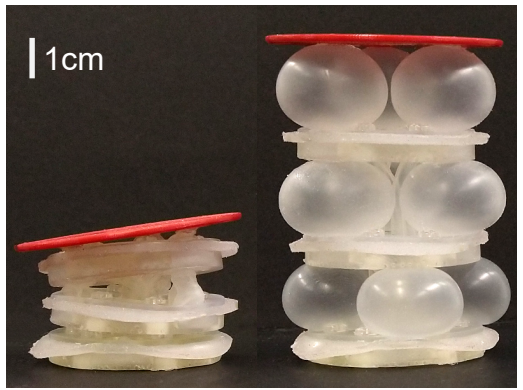

Fig. 1. Implementation of nine HBMAs in a hybrid soft-rigid continuum robot. (left) Relaxed and slightly relaxed actuator; (right) Inflated actuator.

initially flat. Furthermore, the ballooning membranes can be modular parts of structures, e.g., toroidal structures, that provide resistance to buckling from external loads (Fig.1).

The inflation of hyperelastic membranes has been widely studied in material science. From the first characterization of the inflation of a rubber sheet [12], to the modern models of inflated elastomeric membranes in contact with complex shapes or deformable environments [13], [14], most of the proposed models remain too complex to be used in a soft robotic controllers or observers. Indeed since the aim of such models is to describe accurately and locally (at the material level) the behavior of highly nonlinear phenomena, the complexity increases quickly. On the other hand, most of the soft robotics approaches to model ballooning effects rely on Finite Element Modeling [15], [16] which give a good estimate of the deformation, but are computationally expensive and difficult to integrate into robot controllers.

Other ballooning actuators have been proposed in the literature, Baumgartner et al. for instance developed a highspeed ballooning actuator inflated by the contraction of a dielectric elastomeric membrane [17], but the associated model does not take into account the contact at the membrane level. An actuator composed of several hybrid soft-rigid ballooning elements in series and its model were proposed by Overvelde et al. [18]. This model shows how to take advantage of the balloons snap-through instabilities, but is only valid for a closed volume. Finally, Wang et al. proposed a model for the PneuNets actuator to relate the bending moment to the pressure [19]. In particular, the model for these balloons in series takes into account the interaction force between two ballooning elements in contact with each other, but does not apply for a linear ballooning actuator.

Extending on our group's previous soft implantable actuators capable of elongating $36.3 \%$ [20], we propose the design 


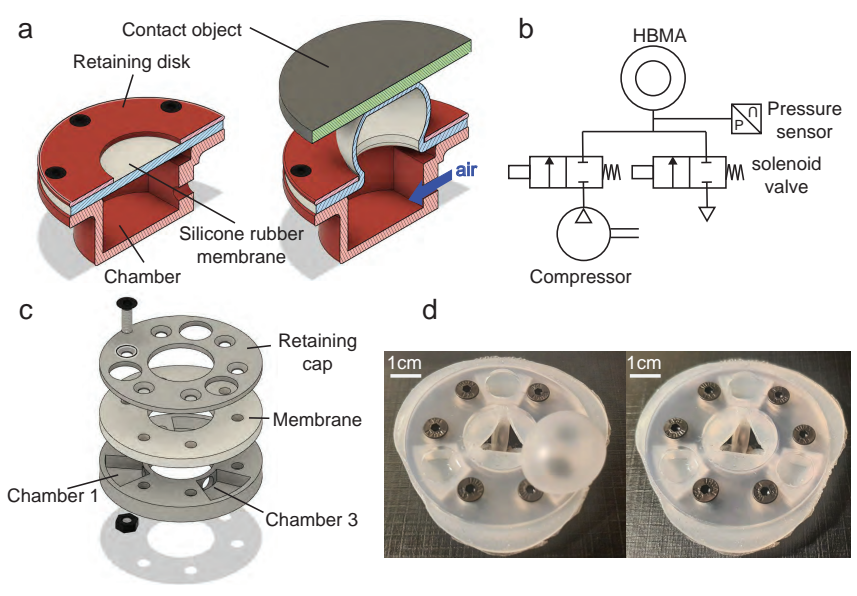

Fig. 2. Hyperelastic Ballooning Membrane Actuator (HBMA). a) Section view of an HBMA interacting with a contacting object b) Pneumatic diagram of the electro-pneumatic circuit used to supply the HBMA c) Implementation of nine HBMAs in a hybrid soft-rigid continuum robot.

and model of a highly extensible and tough actuator: the Hyperelastic Ballooning Membrane Actuator (HBMA). The contributions of this paper include: (1) a model of pressure, membrane height and object interaction of a hyperelastic ballooning membrane controllable by the applied voltage to a micro-compressor (2) easy fabrication of a hyperelastic ballooning membrane actuator by leveraging a hybrid material approach (3) the proposal of a flat actuator which is both highly extensible and compressible.

\section{MATERIAL AND METHODS}

\section{A. Hyperelastic Ballooning Membrane Actuator}

As shown in Fig. 2, the HBMA is composed of a silicone rubber membrane clamped between two rigid parts denoted chamber and retaining disk respectively. For experimentation purposes, the chamber contains a cavity and a port to connect the tubing. On the other hand, the retaining disk consists of a thin rigid disk with a hollow inner circle. Initially flat, the membrane can be deformed by injecting compressed air into the chamber. Thanks to the boundary conditions applied by the retaining disk to the rubber silicone membrane, the latter balloons spherically out of the chamber. The balloon generated by the inflation of the membrane can be used to apply force to the environment or move contacting objects.

To inflate or deflate the HBMA, an electro-pneumatic circuit composed of one micro-compressor, two solenoid valves and a pressure sensor is used. For the case where the compressor only actuates one HBMA, the valve is not used, but it allows the future use of a single compressor for several actuators. The membranes of the HMBAs are obtained by molding platinum-catalyzed silicone Ecoflex 00-30 (SmoothOn, Inc) into 3D printed molds. The two parts of the Ecoflex 00-30 are mixed in equal quantities, then poured into molds and left to cure at room temperature for at least four hours. After unmolding, a post-curing process is performed by heating the membranes in an oven for two hours at $80^{\circ} \mathrm{C}$ and then one hour at $100^{\circ} \mathrm{C}$. The dimensions of the HBMA

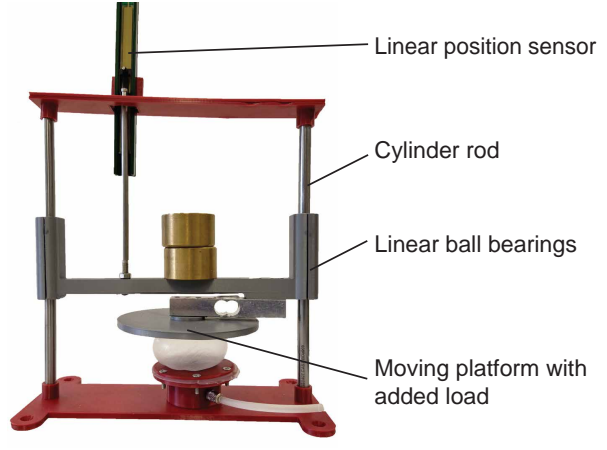

Fig. 3. Characterization setup used to characterize the HBMA and validate the model. The trial illustrated corresponds to the scenario with a $2 \mathrm{~mm}$ membrane, $7.5 \mathrm{~mm}$ inner radius and a $400 \mathrm{~g}$ load.

can be tuned to fit the applications. In particular, the main design parameters are the inner radius of the retaining disk, as well as the membrane thickness. For the model validation, several dimensions of retaining disk inner radius $(7.5,10$, and $12.5 \mathrm{~mm}$ ) and two membrane thicknesses (2 and $3 \mathrm{~mm})$ have been tested. However, these dimensions are scalable to make smaller actuators as shown in the application example described in section II-C.

\section{B. Experimental characterization and model validation}

The characterization aims to measure the displacement and the internal pressure of the HBMA for several loading conditions, compressor speeds, retaining disk inner radii and membrane thicknesses. This experimental characterization also aims to validate the model proposed in the section III. Fig. 3 shows the characterization setup. It consists of a platform and a frame connected with a vertical prismatic joint allowing the platform to freely move in one direction. Using a linear position sensor, the displacement of the platform can be measured. Some calibrated weights can be placed on the moving platform in order to vary the loading condition during the HBMA characterization. During the characterization, an HBMA is placed under the moving platform and its membrane is inflated using the electro-pneumatic circuit. When the HBMA's membrane starts ballooning, it pushes vertically the moving platform and load. After a few seconds, the membrane is deflated by opening the output valve and the platform comes back to its original position. The HBMA inflation is repeated for four different loads $(200,400,600$, and $800 \mathrm{~g}$ ), five compressor speeds $(20 \%, 40 \%, 60 \%, 80 \%$ and $100 \%$ of its nominal speed), two membrane thicknesses (2 and $3 \mathrm{~mm})$ and three inner radii of the retaining disk (7.5, 10 , and $12.5 \mathrm{~mm}$ ), for a total of 150 trials.

\section{Hybrid Soft-Rigid Continuum robot}

Due to the simple design of the HBMA, it is easy to manufacture and can be used in several applications. In this paper, we propose to use it in a hybrid soft-rigid continuum robot. To build such a robot, parallel layers of HBMAs are stacked together as shown in Fig. 1. To miniaturize the size of the robot and simplify the manufacturing and assembly 
process, the dimensions have been reduced, the three retaining disks have been merged into one part, and similarly for the chambers. To simplify the manufacturing process and ensure the consistency of the membrane thickness, the three membranes of each layer have been fabricated as a single silicone part. Once the jointed membrane is clamped between the two rigid 3D printed parts, each chamber can be inflated independently to balloon the membranes to different heights. Finally, to bond the layers, Sil-Poxy (Smooth-on Inc.) silicone adhesive was glued to the center of the membranes, aligned and pressed with the upper layer during setting.

Once assembled, each layer is $9 \mathrm{~mm}$ thick (with a noninflated membrane of $2 \mathrm{~mm}$ ) and with an external diameter of $44 \mathrm{~mm}$. The $17.5 \mathrm{~mm}$ internal diameter of the layer can be used to pass the tubing necessary to inflate the chamber. For the presented continuum robot, the retaining inner radius has been reduced to $4.5 \mathrm{~mm}$. Finally, the weight of a layer is $17.6 \mathrm{~g}$. On the final robot, by inflating the three HBMAs of a layer simultaneously, the next layer is translated along the $\mathbf{z}$ direction of the layer. On the other hand, inflating the three HBMAs differently bends the next layer.

\section{MODELS}

In this section, a model of the HBMA's extension and pressure variation while inflated or deflated is proposed. This model is obtained in three steps. The first step consists of finding the relations between the force, the pressure and the geometry of the membrane at equilibrium. The second step is to model the thermodynamic behavior of the compressed gas in the chamber. Finally, the last step gives the relation between the voltage applied to the compressor and valves, and the mass flow rate entering or exiting the chamber.

\section{A. Pressure and force equilibrium}

The first aim here is to find the relation between $p$ and $F$, respectively the fluid pressure inside the chamber (that pressurizes the membrane) and the interaction force from the object in contact, with the geometry of the deformed inflated membrane. In this paper, the geometry of the deformed membrane under an external load is assumed to follow the shape of a spherical segment. Similarly to the constant curvature assumption for continuum soft robots, this assumption aims to simplify the model and reduce the number of kinematic parameters to three. Therefore, the geometry of the deformed membrane can be fully defined by introducing $r_{1}$ as the inner radius of the retaining disk, $h_{1}$ the height of the virtual nonindented spherical cap, and $h_{2}$ the height of the indented spherical cap. Fig. 4 shows the assumed equivalent free body diagram of the HBMA. This assumption also constrains the contact surface to be parallel to the retaining disk.

Assuming that the ballooning membrane under contact follows a spherical segment, the volume of fluid $V_{f}$ contained in the HBMA can directly by given as:

$$
\begin{aligned}
V_{f}= & V_{0}+\frac{\pi}{6}\left(h_{1}-h_{2}\right)\left(3 r_{1}^{2}+3 h_{2}\left(\frac{h_{1}^{2}+r_{1}^{2}}{h_{1}}-h_{2}\right)\right. \\
& \left.+\left(h_{1}-h_{2}\right)^{2}\right)
\end{aligned}
$$

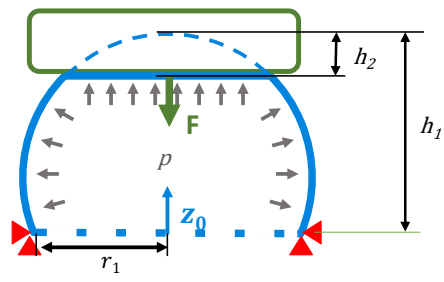

Fig. 4. Free body diagram of an inflated membrane interacting with an external load F (plane contact)

where $V_{0}$ is the dead volume in the pipe and chamber. Assuming equilibrium at the contact level between the object and the membrane yields:

$$
F=\pi h_{2}\left(\frac{h_{1}^{2}+r_{1}^{2}}{h_{1}}-h_{2}\right) p
$$

where $p$ denotes the relative pressure (to the environment) not the absolute pressure. It is also assumed that the pressure applied to the membrane is homogeneous.

The silicone membrane is assumed to be an incompressible material with an equi-biaxial extension (due to the symmetry of revolution). Therefore, the principal stretches can be computed as:

$$
\lambda=\lambda_{1}=\lambda_{2}=\frac{1}{\sqrt{\lambda_{3}}}=\sqrt{\frac{h_{1}^{2}+r_{1}^{2}-h_{2}^{2}}{r_{1}^{2}}}
$$

where $\lambda_{1}$ and $\lambda_{2}$ are the principal stretches along the two tangential directions of the membrane and $\lambda_{3}$ is the stretch normal to the membrane. For simplicity, the tangential stretches will from now on be referred to as $\lambda$.

The second aim in this paper is to compare two hyperelastic models: the Neo-Hookean (NH) model and the MooneyRivlin (MR) model. Their respective strain energy functions are defined as follows (under an equi-biaxial extension assumption):

$$
\begin{gathered}
W_{N H}=\frac{E}{6}\left(2 \lambda^{2}+\frac{1}{\lambda^{4}}-3\right) \\
W_{M R}=C_{1}\left(2 \lambda^{2}-\frac{1}{\lambda^{4}}-3\right)+C_{2}\left(\frac{2}{\lambda^{2}}+\lambda^{4}-3\right)
\end{gathered}
$$

where $E$ is the equivalent NH Young's modulus, and $C_{1}$ and $C_{2}$ are the MR coefficients. The total potential energy $E_{p}$ of the HBMA can then be written as follows:

$$
E_{p}=\int_{V_{m}} W d V-\int_{V f} p d V-F\left(h_{1}-h_{2}\right)
$$

where $V_{m}$ is the volume of the membrane and $W$ can be replaced by either of the strain energy functions given in (4) and (5). Due to the assumption of incompressibility of the membrane, $V_{m}$ is constant and can be computed as:

$$
V_{m}=t_{0} \pi r_{1}^{2}
$$

where $t_{0}$ is the initial membrane thickness (before deformation). 
Finally, by applying the principle of minimum potential energy to (6), the pressure as a function of $h_{1}$ and $h_{2}$ can be expressed as:

$$
p=f(h 1, h 2)
$$

where $f$ is a nonlinear function. The expression of $f$ is also dependent on whether the NH or the MR strain energy function is used.

\section{B. Thermodynamic and dynamic models}

Following on from the pressure model, the aim here is to introduce the dynamic behavior, when the air is injected by the compressor in the chamber, or when the output valve is opened. In particular, the objective of this section is to find the relation between the time derivatives of the two state variables $h_{1}$ and $h_{2}$ and the mass flow rate of the fluid entering or exiting the chamber.

To start, the assumption that the air in the chamber is behaving as a perfect gas, yields:

$$
\left(p+p_{\text {env }}\right) V=m R T
$$

where $p_{e n v}$, the absolute pressure of the environment (atmospherique pressure), is added to $p$ to obtain the absolute pressure in the chamber, and $R$ is the specific gas constant. Then differentiating (9) leads to:

$$
\frac{d p}{d t} V T+\frac{d V}{d t}\left(p+p_{\text {env }}\right) T+\frac{d T}{d t}\left(p+p_{\text {env }}\right) V=q_{m} R T^{2}
$$

where $q_{m}$ is the mass flow rate of gas entering (or exiting if negative) the chamber.

Combined with the assumption that the temperature in the chamber is constant, (10) can be simplified to:

$$
\frac{d p}{d t}=\frac{1}{V}\left(q_{m} R T-\left(p+p_{\text {env }}\right) \frac{d V}{d t}\right)
$$

The total time derivative of the volume and pressure can be written as follows:

$$
\begin{gathered}
\frac{d V_{f}}{d t}=\frac{\partial V_{f}}{\partial h_{1}} \dot{h_{1}}+\frac{\partial V_{f}}{\partial h_{2}} \dot{h_{2}} \\
\frac{d p}{d t}=\frac{\partial p}{\partial h_{1}} \dot{h_{1}}+\frac{\partial p}{\partial h_{2}} \dot{h_{2}}
\end{gathered}
$$

where $p$ and $V_{f}$ can be replaced by the expression obtained in (8) and (1) respectively.

Assuming a quasi-static behavior, the variation of force over time can be obtained from (2):

$$
\begin{aligned}
\dot{F}= & \frac{\pi}{h_{1}^{2}}\left(\left(h_{2}\left(h_{1}^{2}-r_{1}^{2}\right) \dot{h_{1}}+h_{1}\left(r_{1}^{2}+h_{1}^{2}-2 h_{1} h_{2}\right) \dot{h_{2}}\right) p\right. \\
& \left.+h_{1} h_{2}\left(r_{1}^{2}+h_{1}^{2}-h_{1} h_{2}\right) \dot{p}\right)
\end{aligned}
$$

The time derivative of the two state variables $h_{1}$ and $h_{2}$ can be expressed as a function of $h_{1}, h_{2}, q_{m}$, and $F$ by

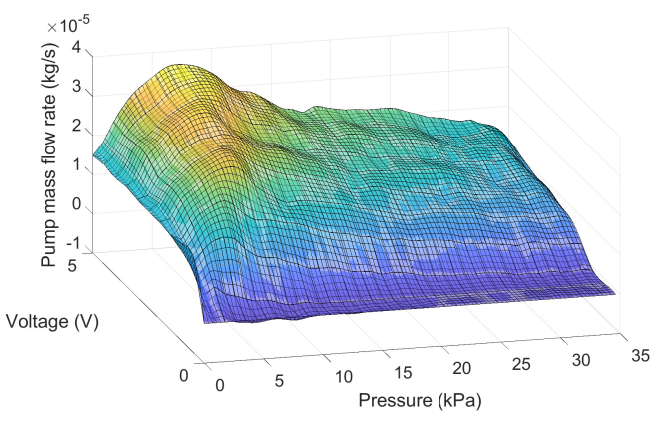

Fig. 5. Experimental compressor characterization

solving the system of equations (13) and (14) and substituting the variables with those previously obtained:

$$
\begin{aligned}
& \dot{h_{1}}=g_{1}\left(h_{1}, h_{2}, q, \dot{F}\right) \\
& \dot{h_{2}}=g_{2}\left(h_{1}, h_{2}, q, \dot{F}\right)
\end{aligned}
$$

where $g_{1}$ and $g_{2}$ are two nonlinear functions.

Finally, knowing the mass flow rate entering the chamber and the variation of force applied to the membrane, the shape of the membrane together with the pressure inside the chamber can be computed by numerical integration. In this paper, the Euler method (Matlab/Simulink ode1) was used to perform the numerical integration.

\section{Compressor and valves}

To complete the model, the relation between the gas mass flow rate given by the compressor and both the voltage applied to the latter and the pressure in the chamber still remain to be defined. In this paper, an experimental characterization of the compressor was carried out. This characterization is illustrated in Fig. 5. The valves have been similarly characterized to give the relation between the pressure in the chamber and the mass flow rate.

In the simulations presented in section IV, the fluid mass flow rate is interpolated from the characterization surface shown in Fig. 5 and the equivalent curve for the valve. The characterization of the compressor was stopped at $35 \mathrm{kPa}$ due to the sensor saturation.

\section{RESUlts}

\section{A. Equilibrium load and pressure}

Fig. 6 shows the relation between the equilibrium pressure and load for different values of $h_{1}$ and $h_{2}$. These surfaces are obtained from (8) and (2) with the equilibrium load computed by dividing the contact force by the gravitational acceleration.

For small values of $h_{1}$, the difference between the NH model and MR models is not very significant, but the difference increases with $h_{1}$. Also, for higher ratios of $h_{1} / h_{2}$, the equilibrium load gradient along $h_{2}$ becomes constant. This means that for highly inflated HBMAs, the relation between the equilibrium load and the indented height could be linearized, which would simplify even more the model. 

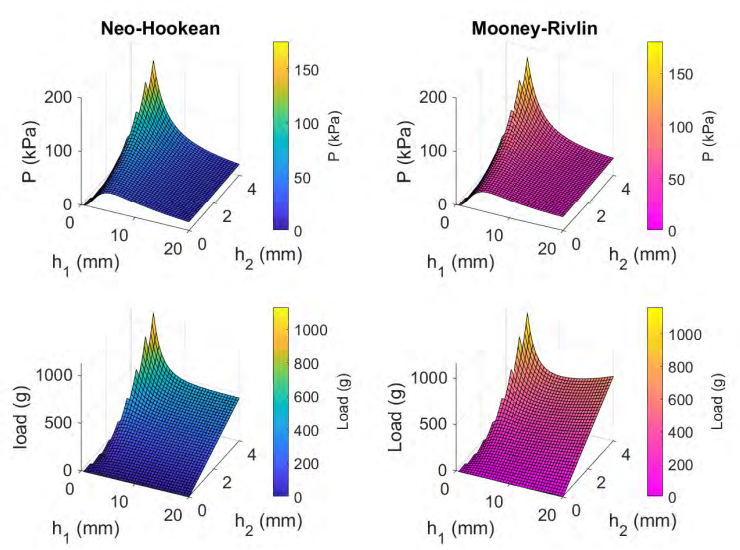

Fig. 6. Equilibrium pressure and load as a function of $h_{1}$ and $h_{2}$ for a thickness of the membrane $t_{0}=3 \mathrm{~mm}$, and a retaining inner radius $r_{1}=7.5 \mathrm{~mm}$. The left surfaces are the ones obtained with the NH model. The right surfaces are for MR material model

TABLE I

MODEL PARAMETERS USED FOR THE SIMULATION

\begin{tabular}{c|c|c|c}
\hline \hline Notation & Description & Value & Unit \\
\hline$V_{0}$ & Dead volume in the chamber & 3 & $\mathrm{~cm}^{3}$ \\
$E$ & Equivalent Young Modulus of the NH & 130.6 & $\mathrm{kPa}$ \\
& model & & \\
$C_{1}$ & First MR coefficient & 21.8 & $\mathrm{kPa}$ \\
$C_{2}$ & Second MR coefficient & 600 & $\mathrm{~Pa}$ \\
$R$ & Specific gas constant for dry air & 287.058 & $\mathrm{Jkg}^{-1} \mathrm{~K}^{-1}$ \\
$T$ & Temperature in the chamber & 293.15 & $\mathrm{~K}$ \\
$p_{\text {env }}$ & Pressure of the environment of the & 100.0 & $\mathrm{kPa}$ \\
& HBMA & \\
\hline \hline
\end{tabular}

\section{B. Model validation}

To validate the model proposed in section III, data was collected following the protocol described in section II-B and compared with a Matlab/Simulink model computing the state variables $h_{1}, h_{2}$ and $P$, knowing the voltage input of the compressor, the voltage input of the valves and the load applied to the HBMA. Since the experimental setup can measure only the height of the membrane, $h=h_{1}-h_{2}$ will be computed to compare the model and the experimental results. The parameters used in the simulation are given in Table I. The lack of characterization of the Ecoflex-0030 hyperelastic material parameters for an equi-biaxial loading in the literature lead us to tune the parameters $E$ for the $\mathrm{NH}$, and $C_{1}$ and $C_{2}$ for the Mooney Rivlin around the value published for uniaxial load [21]. After tuning, these parameters have been kept constant for all of the simulations in the rest of the paper.

Fig. 7 shows the results obtained for the five inflations (at different compressor speeds) of the HBMA with a membrane thickness of $3 \mathrm{~mm}$ and an inner radius of $12.5 \mathrm{~mm}$ without any load. The figure shows that the two models give different results, particularly for the pressure. From Fig. 7, it can be observed that the models give a better estimate for small inflations. This can be explained by the fact that the model is open-loop and is integrated on the positions and pressure obtained in the previous time steps.

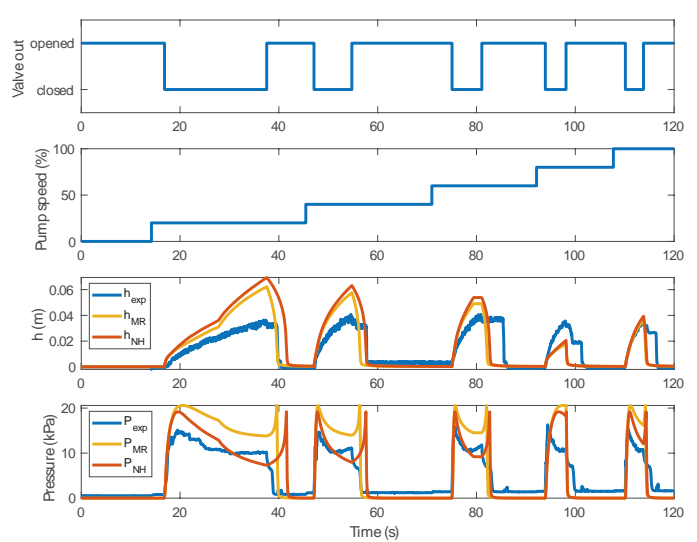

Fig. 7. Experimental and simulation results for an HBMA without load, with a retaining inner radius of $12.5 \mathrm{~mm}$ and membrane thickness of $3 \mathrm{~mm}$.

To compare the accuracy of the two models across several loads, retaining disk inner radii, and membrane thicknesses tried, the Normalized Root Mean Squared Deviation (NRMSD) was computed for each parameter. The NRMSD was chosen as it is well-suited to compare trials with different ranges of measured variables, as was computed as follows:

$$
N R M S D=\frac{\sqrt{\frac{\sum(\hat{y}-y)^{2}}{N}}}{y_{\max }-y_{\min }}
$$

where $y$ is the measured variable, $\hat{y}$ is the estimated variable, $N$ the number of compared data points and $y_{\max }$ and $y_{\min }$ are respectively the highest and the lowest values measured.

Fig. 8 shows the comparison of the NRMSD obtained for the NH and the MR models. Out of the 30 scenarios tested, the simulation diverged in three cases and only for the $\mathrm{NH}$ model. The three cases are for the smallest retaining inner radius and with the highest loads. For clarity of the results, the data points related to these cases have been removed from Fig. 8 (as the NRMSD was much greater than 1). Fig. 8 shows that despite being less robust, the $\mathrm{NH}$ model is on average more accurate. In particular, the main differences can be seen for the pressure. This can be explained by the model obtained with the MR strain energy function seeming to be more sensitive to sudden variations of mass flow rate, also impacting the accuracy of the computed force. This difference is further illustrated in Fig. 9, where a peak in the computed force is visible when the output valve is opened for the MR model.

\section{Application: Hybrid Soft-Rigid Continuum robot}

Fig. 10 shows how the HBMA can be used in a hybrid soft-rigid continuum soft robot. The robot is actuated sequentially generating the displacement and rotation of the layers. Without reaching the limit (which would mean breaking the membrane) the average angle between one layer and the next one, as well as the displacement are summarized in Table II. The maximum displacement and rotation of the robot's endeffector can be further increased by stacking more layers.

Fig. 10 also shows that the model previously presented can be used to estimate the height of each HBMA sequentially 


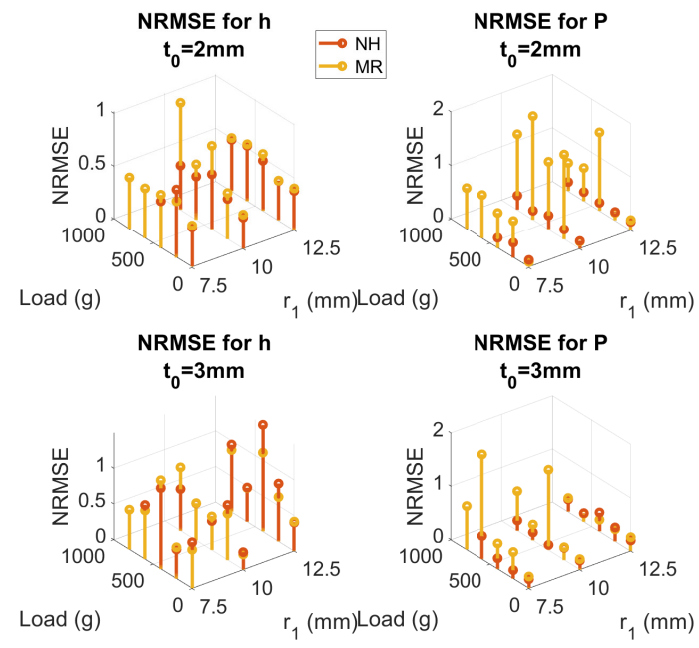

Fig. 8. Normalized Root Mean Squared Deviation (NRMSD) for the estimated pressure and height.

TABLE II

AVERAGE ANGLE AND DISPLACEMENT BETWEEN TWO LAYERS

\begin{tabular}{c|c|c|c}
\hline \hline $\begin{array}{c}\text { Number of HBMA } \\
\text { inflated }\end{array}$ & angle (deg) & length (mm) & extension (\%) \\
\hline 0 & 2.3 & 9.6 & 0 \\
1 & 28.9 & 18.0 & 88 \\
2 & 28.5 & 23.0 & 140 \\
3 & 1.3 & 26.7 & 179 \\
\hline \hline
\end{tabular}

actuated. From the estimated displacement, the position and orientation of the last layer could easily be computed using a standard forward kinematic approach for parallel robots.

\section{Discussion AND CONCLUSION}

In this paper, we proposed the model and validation of a hybrid soft-rigid actuator based on a ballooning membrane. Thanks to assumptions taken on the deformed geometry of membrane, the proposed model remains simple and could be used to develop controllers or observers for ballooning soft robots. In particular, two hyperelastic models have been tested and the parameters of these material models could be tuned to fit more accurately once the design is fixed. Additionally, a trade-off between accuracy and robustness
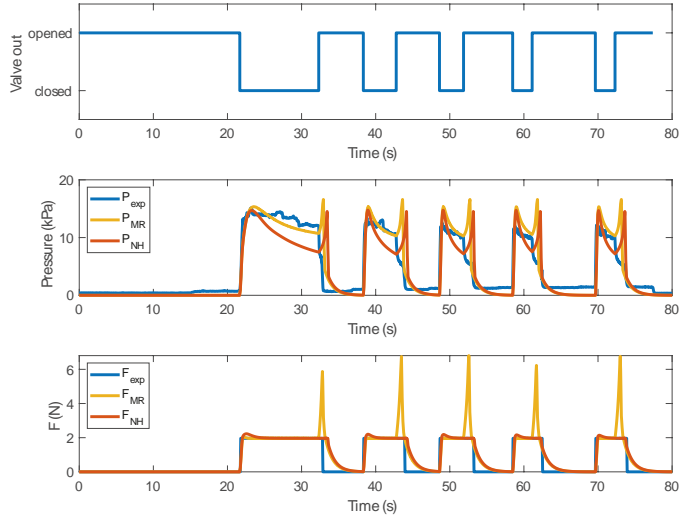

Fig. 9. Experimental and simulation results for an HBMA with a retaining disk inner radius of $12.5 \mathrm{~mm}$, membrane thickness of $2 \mathrm{~mm}$ and a load of $200 \mathrm{~g}$.

needs to be made while choosing the hyperelastic model. Indeed, in our study we pointed out that when the MR model is used, the model is more robust but less accurate than when the NH behavior is assumed. Other hyperelastic models such as Gent, Ogden or Yeoh remain to be investigated and could potentially increase the accuracy and robustness.

To conclude, the HBMA shows high extensibility and great resistance to an external load. Taking advantage of its thin overall thickness, we have shown through experimental characterization that the actuator can reach an extensibility higher than $179 \%$ and stand more than 20 times its weight without buckling. Finally, its flat design when uninflated seems suitable for the application inside the body. In future work, we aim to test the ability of the HBMA to perform in more clinically realistic scenarios. Using the proposed model, the development of controllers and observers will be further investigated, as well as the integration of sensing capabilities to give more autonomy to the hybrid soft-rigid continuum ballooning robot.

\section{ACKNOWLEDGEMENT}

The authors would like to thank Mohammad Bahgat for his help with the video montage.

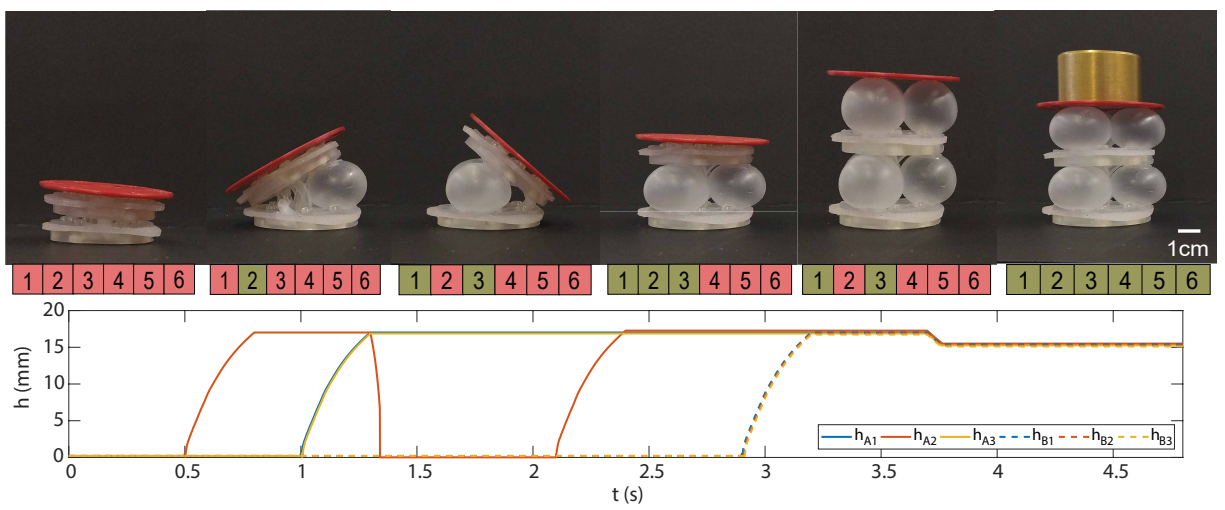

Fig. 10. Continuum hybrid soft-rigid robot. a) All the chambers are at environment pressure. b) Chambers 1 and 3 are inflating, while chamber 2 remains closed. c) Chamber 1 is deflating while chambers 1 and 2 are closed. 


\section{REFERENCES}

[1] N. Agharese, T. Cloyd, L. H. Blumenschein, M. Raitor, E. W. Hawkes, H. Culbertson, and A. M. Okamura, "Hapwrap: Soft growing wearable haptic device," in 2018 IEEE International Conference on Robotics and Automation (ICRA). IEEE, 2018, pp. 1-5.

[2] D. D. Damian, K. Price, S. Arabagi, I. Berra, Z. Machaidze, S. Manjila, S. Shimada, A. Fabozzo, G. Arnal, D. Van Story, et al., "In vivo tissue regeneration with robotic implants," Science Robotics, vol. 3, no. 14, p. eaaq0018, 2018.

[3] E. T. Roche, M. A. Horvath, I. Wamala, A. Alazmani, S.-E. Song, W. Whyte, Z. Machaidze, C. J. Payne, J. C. Weaver, G. Fishbein, et al., "Soft robotic sleeve supports heart function," Science translational medicine, vol. 9, no. 373, 2017.

[4] L. N. Awad, J. Bae, K. O'donnell, S. M. De Rossi, K. Hendron, L. H. Sloot, P. Kudzia, S. Allen, K. G. Holt, T. D. Ellis, et al., "A soft robotic exosuit improves walking in patients after stroke," Science translational medicine, vol. 9, no. 400, 2017.

[5] P. Moshayedi, G. Ng, J. C. Kwok, G. S. Yeo, C. E. Bryant, J. W. Fawcett, K. Franze, and J. Guck, "The relationship between glial cell mechanosensitivity and foreign body reactions in the central nervous system," Biomaterials, vol. 35, no. 13, pp. 3919-3925, 2014.

[6] C. Huang, J. Holfeld, W. Schaden, D. Orgill, and R. Ogawa, "Mechanotherapy: revisiting physical therapy and recruiting mechanobiology for a new era in medicine," Trends in molecular medicine, vol. 19, no. 9, pp. 555-564, 2013.

[7] M. Atwya, C. Kavak, E. Alisse, Y. Liu, and D. Damian, "Flexible and expandable robot for tissue therapies-modeling and design," IEEE Transactions on Biomedical Engineering, 2020.

[8] P. F. M. Pinheiro, A. C. S. e Silva, and R. M. Pereira, "Current knowledge on esophageal atresia," World journal of gastroenterology: $W J G$, vol. 18 , no. 28, p. $3662,2012$.

[9] J. E. Foker, B. C. Linden, E. M. Boyle Jr, and C. Marquardt, "Development of a true primary repair for the full spectrum of esophageal atresia." Annals of surgery, vol. 226, no. 4, p. 533, 1997.

[10] F. Connolly, C. J. Walsh, and K. Bertoldi, "Automatic design of fiberreinforced soft actuators for trajectory matching," Proceedings of the National Academy of Sciences, vol. 114, no. 1, pp. 51-56, 2017.

[11] M. Cianchetti, T. Ranzani, G. Gerboni, T. Nanayakkara, K. Althoefer, P. Dasgupta, and A. Menciassi, "Soft robotics technologies to address shortcomings in today's minimally invasive surgery: the stiff-flop approach," Soft robotics, vol. 1, no. 2, pp. 122-131, 2014.

[12] L. R. G. Treloar, "Strains in an Inflated Rubber Sheet, and the Mechanism of Bursting," Rubber Chemistry and Technology, vol. 17, no. 4, pp. 957-967, 121944.

[13] W. W. Feng and H. Pangnan, "On the general contact problem of an inflated nonlinear plane membrane," International Journal of Solids and Structures, vol. 11, no. 4, pp. 437 - 448, 1975.

[14] N. Kumar and A. DasGupta, "On the contact problem of an inflated spherical hyperelastic membrane," International Journal of NonLinear Mechanics, vol. 57, pp. 130 - 139, 2013.

[15] A. Rodríguez, E. Coevoet, and C. Duriez, "Real-time simulation of hydraulic components for interactive control of soft robots," in 2017 IEEE International Conference on Robotics and Automation (ICRA), 2017, pp. 4953-4958.

[16] Y. Elsayed, A. Vincensi, C. Lekakou, T. Geng, C. M. Saaj, T. Ranzani, M. Cianchetti, and A. Menciassi, "Finite element analysis and design optimization of a pneumatically actuating silicone module for robotic surgery applications," Soft Robotics, vol. 1, no. 4, pp. 255-262, 2014.

[17] R. Baumgartner, A. Kogler, J. M. Stadlbauer, C. C. Foo, R. Kaltseis, M. Baumgartner, G. Mao, C. Keplinger, S. J. A. Koh, N. Arnold, Z. Suo, M. Kaltenbrunner, and S. Bauer, "A lesson from plants: Highspeed soft robotic actuators," Advanced Science, vol. 7, no. 5, p. 1903391, 2020.

[18] J. T. B. Overvelde, T. Kloek, J. J. A. D'haen, and K. Bertoldi, "Amplifying the response of soft actuators by harnessing snap-through instabilities," Proceedings of the National Academy of Sciences, vol. 112, no. 35, pp. 10863-10868, 2015.

[19] J. Wang, Y. Fei, and W. Pang, "Design, modeling, and testing of a soft pneumatic glove with segmented pneunets bending actuators," IEEE/ASME Transactions on Mechatronics, vol. 24, no. 3, pp. 9901001, 2019.

[20] E. Perez-Guagnelli, J. Jones, A. H. Tokel, N. Herzig, B. Jones, S. Miyashita, and D. D. Damian, "Characterization, simulation and control of a soft helical pneumatic implantable robot for tissue regeneration," IEEE Transactions on Medical Robotics and Bionics, vol. 2, no. 1, pp. 94-103, 2020.

[21] L. Marechal, P. Balland, L. Lindenroth, F. Petrou, C. Kontovounisios, and F. Bello, "Toward a common framework and database of materials for soft robotics," Soft Robotics, vol. 0, no. 0, p. null, 0, pMID: 32589507. 